\title{
Premature ovarian insufficiency - aetiopathology, epidemiology, and diagnostic evaluation
}

\author{
Ewa Rudnicka, Jagoda Kruszewska, Klaudia Klicka, Joanna Kowalczyk, Monika Grymowicz, \\ Jolanta Skórska, Wojciech Pięta, Roman Smolarczyk
}

Department of Gynaecological Endocrinology, Medical University of Warsaw, Warsaw, Poland

\begin{abstract}
Premature ovarian insufficiency (POI) is defined as a cessation of ovarian function before the age of 40 years. It is associated with hypoestrogenism and loss of residual follicles, both of which lead to menstrual abnormalities, pregnancy failures, and decreased health-related quality of life. The prevalence of POI is estimated at $1 \%$ in the general population. Current European Society of Human Reproduction and Embryology (ESHRE) diagnostic criteria include: amenorrhoea or oligomenorrhoea for at least four months and increased follicle-stimulating hormone (FSH) levels > $25 \mathrm{IU} / \mathrm{l}$ measured twice (with a four-week interval). The aetiopathogenesis of the disease in most cases remains unexplained. Nevertheless, in some patients with POI, genetic abnormalities, metabolic disorders, autoimmunity, iatrogenic procedures, infections, or environmental factors have been established as underlying causes of the syndrome.
\end{abstract}

Key words: premature ovarian insufficiency, Turner syndrome, autoimmunity.

\section{Introduction}

Premature ovarian insufficiency (POI) is a disorder characterised by ovarian dysfunction leading to hypoestrogenism and loss of residual follicles in the gonads associated with oligo- or amenorrhoea and subfertility or infertility. Among women with primary amenorrhoea, the frequency of $\mathrm{POI}$ is $10-28 \%$ and in those with secondary amenorrhoea 4-18\% [1].

First case report originates from 1942 by Fuller Albright, who termed the condition "primary ovarian insufficiency" [2]. Later it was recognised under several other names, such as "premature ovarian failure" or "premature menopause". The two latter expressions were too distressing for the patients, stigmatising irreversible failure and indicating a severe condition with complete follicular exhaustion [3]. New European Society of Human Reproduction and Embryology (ESHRE) guidelines propose the term "premature ovarian insufficiency" for use in research and clinical practice [4].

\section{Epidemiology}

The risk of premature ovarian insufficiency (POI) before the age of 40 years is $1 \%$ [5]. The prevalence of menopause varies according to age, and it is $1: 10,000$ at the age of 18-25 years, $1: 1000$ in women aged
$25-30$ years, and $1: 100$ in the age range $35-40$ years. POI is related with familiar occurrence in about $15 \%$ of cases, which suggests a genetic aetiological background [6, 7]. Epidemiological studies have shown differences in the occurrence of POI depending on ethnicity, and it is highest in Caucasian, African American, and Hispanic women [8].

\section{Aetiopathology}

The aetiopathology of premature ovarian insufficiency in the majority of cases is unknown and is termed as spontaneous or idiopathic POI [5, 9]. Some underlying reasons for POI have been established. Most authors group them considering the following aspects: genetic abnormalities, metabolism disorders, autoimmunity, iatrogenic procedures, infections, and environmental factors.

\section{Genetic abnormalities}

Genetic causes are responsible for $10.8 \%$ of POI [10] and can be divided into two main groups: chromosomal and gene abnormalities.

Turner syndrome, X-monosomy, is the most common abnormality in the first group. Clinically, it manifests with primary amenorrhoea, short stature, and characteristic phenotypic features like webbing of the 
neck or cubitus valgus. The gonads are dysgenetic and consist of fibrous tissue. This condition illustrates that normal ovarian function requires two efficient $X$ chromosomes [10, 11].

On the other hand, the presence of $X$ trisomy may also result in ovarian dysfunction $[12,13]$. Gene abnormalities can pertain to mutation on $\mathrm{X}$ as well as on autosomal chromosomes.

The most common is FMR1 premutation located on $X$ chromosome. The mutation rests upon increased expansion of CGG triplets in the untranslated region. Patients with FMR1 premutation (CGG present 55-199 times) are at increased risk of developing POI [12]. The incidence of ovarian dysfunction among carriers reaches $12.9 \%$ [14]. Full mutations characterised by more than 200 repetitions inactivate the gene by methylation and lead to fragile $X$ syndrome, and are also the most common inherited cause of disability among men [15].

Other important genes, mutations of which lead to $\mathrm{POI}$, are BMP-15, located within X chromosome, active in the process of folliculogenesis [16], and autosomal GD9, an oocyte-secreted growth factor that has impact on differentiation of oocyte, granulosa, and theca cells $[17,18]$.

Less common genes include: XIST ( $X$ inactivationspecific transcript) involved in inactivation of one of the $X$ chromosomes, POLG (mitochondrial DNA polymerase $\gamma$ ), CENPI, PGMRC1, AR, FOXO4, AGTR2, BHLHB9, FSHR, GNAS, FOXL2, GALT, AIRE, STAR, CYP17A1, CYP19A1, eIF2B, NOG, $A T M, P M M 1$, and BMPR1B $[10,14]$.

\section{Aułoimmunity}

Autoimmune aetiology is reported to have been found in up to $30 \%$ cases of POI and may be present exclusively or can coexist with other autoimmune diseases, including endocrine disorders (mainly adrenal insufficiency, Hashimoto's thyroiditis, and type 1 diabetes mellitus), as well as those non-endocrine (Sjögren's syndrome, rheumatoid arthritis, systemic lupus erythematosus, inflammatory bowel diseases, multiple sclerosis, myasthenia gravis, and alopecia) [19]. Ovarian dysfunction may be a component of autoimmune polyglandular syndromes (APS). APS type 1 is characterised by hypoparathyroidism, adrenal insufficiency, chronic mucocutaneous candidiasis, and the prevalence of POI in $15 \%$ of cases. Type 2 is associated with adrenal insufficiency, autoimmune thyroid disease, type I diabetes mellitus, and POI with a frequency of $3.6-10 \%[19,20]$.

Although the coexistence of autoimmune disorders is frequent in $\mathrm{POI}$, histological examinations on ovarian tissue have documented that signs of oophoritis can be detected only in women with circulating adrenal or ovarian autoantibodies directed to steroidogenic enzymes (steroid-cell autoantibodies - SCA) with a prevalence of $4-5 \%[19,21]$.

\section{Impaired metabolism}

Metabolic disorders leading to $\mathrm{POI}$ include $17-\mathrm{OH}$ deficiency and classic galactosaemia. The latter is associated with mutation of the GALT gene, leading to impaired function of the enzyme called galactose-1-phosphate uridylotransferase, which is significant in the metabolism of galactose. POI seems to be a long-term complication developing in more than $90 \%$ of patients with galactosaemia. Its possible mechanisms include toxicity of galactose metabolites or defective glycosylation of proteins or lipids within ovarian tissue throughout life. The fact that POI develops despite life-long dietary restriction and neonatal diagnosis suggests the start of the toxicity in the prenatal period [22].

17-OH deficiency actually belongs to the group of disorders of sexual differentiations, coexisting with either $46, \mathrm{XX}$ or $46, \mathrm{XY}$ karyotype. The enzymatic defect pertains here to CYP17A1, which shares the activity of steroid $17 \alpha$-hydroxylase and 17-20-lyase and is physiologically active in adrenals and gonads. The block precludes synthesis of all 19-carbon steroids, including oestrogens, thus providing a clinical picture of POI [23].

\section{latrogenic causes}

latrogenic impairment of gonadal tissue mostly pertains to oncologic patients, who are exposed to radiation or chemotherapeutic agents. The risk that oncological treatment will lead to POI increases with age after puberty, with various high-dose chemotherapy regimens and with combined chemo- and radiation therapy [24]. Combination chemotherapy and the use of alkylating agents have been shown to be most gonadotoxic in childhood and in adulthood. Lower risk is associated with antimetabolites, anthracyclic antibiotics, and vinca alkaloids [10]. During radiotherapy a dosage over 9 Gy carries high risk of ovarian insufficiency [24]. Surgery may also contribute to POI, directly because of excision of ovaries or during operations within the pelvis resulting in diminished blood supply to gonads. Laparoscopic excision of bilateral endometriomas leads in $2.4 \%$ of cases to premature ovarian insufficiency, and ovarian endometrioma surgery is associated with a decline in serum $\mathrm{AMH}$ and diminished ovarian reserve [25].

\section{Infections}

There are two main viruses that are suspected as a cause of POI. The first is epidemic parotitis virus, which leads to mumps oophoritis, which can be complicated by ovarian failure. However, most women regain normal function of the gonads after recovery. The second agent is human immunodeficiency virus, which as well as the antiretroviral treatment may impair ovarian function. Moreover, there are also some associations reported between 
POI and viral or microbial infections, such as tuberculosis, varicella, cytomegalovirus, or malaria, but their contribution to pathogenesis remains uncertain $[14,26]$.

\section{Environmental factors}

A recent meta-analysis indicates that environmental pollutants and toxins may also play a role in the pathogenesis of POI. Their impact on the ovaries may be disruptive in multiple ways that can often coexist. The substances that may be significant are: Bisphenol A (which is used for food packing as a component of plastic), polycyclic aromatic hydrocarbons, polychlorinated biphenyls, pesticides, dioxins, genistein, or cigarette smoke. Nevertheless, there is no clear evidence of an environmental impact on $\mathrm{PO}$ except for studies on cigarette smoking [27].

\section{Course of the disease and symptoms}

About $76 \%$ of patients with $\mathrm{POI}$ maintain regular menses in puberty and adulthood, followed by disruptions in cycles later on $[28,29]$. Ovarian function in POI can be intermittent and unpredictable, even followed by spontaneous ovulations in $20 \%$, and conceptions in about $5-10 \%$ of women [3]. Among the clinical symptoms, we can distinguish those that are characteristic for decreased concentration of oestrogens in the blood and occur during menopause: hot flushes, night sweats, vaginal symptoms, dyspareunia, vaginal dryness, sleep disturbance, mood changes, low concentration, dry eyes, altered urinary frequency, low libido, and lack of energy [10]. However, these symptoms do not occur in all women and are less frequent in the case of primary amenorrhoea, implying that these symptoms are due to oestrogen withdrawal rather than oestrogen deficiency [30]. The symptoms can be transient or intermittent and may be variable in severity, because of fluctuations in ovarian activity during spontaneous onset of POI. POI substantially decreases health-related quality of life of affected patients due to increased cardiovascular risk, reduced bone mineral density leading to osteoporosis, and atrophic changes in the genitourinary system. The disease has an undeniably negative impact on the psychological well-being of patients, associated with pregnancy failures and lower satisfaction from sexual life.

\section{Diagnostic evaluation}

Criteria for POI diagnosis are the occurrence of clinical symptoms, amenorrhoea, or rare menstruation for at least four months and increased FSH levels > $25 \mathrm{IU} / \mathrm{I}$ (repeated at four-week intervals) [3]. The FSH serum level is the gold standard test in POI diagnosis. Anti-Müllerian hormone (AMH) may be interpreted only together with the FSH and oestrogen levels. In cases of secondary amenorrhoea, it is necessary to exclude pregnancy by examining the serum beta-HCG (human chorionic gonadotropin) level and to check hormone levels of thyroid-stimulating hormone (TSH) and prolactin, because endocrine diseases can lead to menstrual disorders [29]. It is recommended that chromosomal analysis be performed in all women with POI, and in cases of incidence of $Y$ chromosome gonadectomy should be advised, because of elevated risk of developing gonadal neoplasia (10-30\%) [28, 31]. Additionally, for all women with $\mathrm{POI}$ and their families it is important to carry out fragile- $X$ premutation testing [32]. Due to the fact that some POI cases have an autoimmune background and $\mathrm{POI}$ is one of the diseases that occur in APS, it is important to exclude other autoimmune diseases. According to ESHRE, it is necessary to assess levels of adrenal antibodies $(21 \mathrm{OH}-\mathrm{Ab})$ and thyroid antibodies (TPO-Ab). If results are positive, monitoring of the function of these endocrine glands is needed [4].

\section{Conclusions}

Premature ovarian insufficiency remains a severe medical problem, significantly affecting the patient's life. The aetiopathology of POI in most cases remains unexplained. Recently growing evidence of genetic background has revealed new mutations, which may be the cause of a condition known so far as "idiopathic POI". Diagnosis and evaluation of POI, apart from karyotype, covers exclusion of other conditions leading to amenorrhoea, such as pregnancy, thyroid gland diseases, or hyperprolactinaemia. An important part of the evaluation is assessment of the possibility of autoimmune diseases that may accompany premature ovarian failure.

\section{Disclosure}

The authors report no conflict of interest.

\section{References}

1. Anastasi JN. Premature ovarian failure: an update. Fertil Steril 1998; 70: 1-15.

2. Albright F, Smith PH, Fraser R. A syndrome characterized by primary ovarian insufficiency and decreased stature: report of 11 cases with a digression on hormonal control of axillary and pubic hair. Am J Med Sci 1942; 204: 625-648.

3. Komorowska B. Autoimmune premature ovarian failure. Menopause Rev 2016; 15: 210-214

4. Webber L, Davies M, Anderson R, Bartlett J, Braat D, et al. ESHRE Guideline: management of women with premature ovarian insufficiency. Hum Reprod 2016; 31: 926-937.

5. Coulam CB, Adamson SC, Annegers JF. Incidence of premature ovarian failure. Obstet Gynecol 1986; 67: 604-606.

6. Franic-Ivanisevic M, Franic D, Ivovic M, Tancic-Gajic M, Marina L, Barac M, et al. Genetic Etiology of Primary Premature Ovarian Insufficiency. Acta Clin Croat 2016; 55: 629-635. 
7. Cramer DW, Xu H. Predicting age at menopause. Maturitas 1996; 23: 319-326.

8. Luborsky JL, Meyer P, Sowers MF, et al. Premature menopause in a multi-ethnic population study of the menopause transition. Hum Reprod 2003; 18: 199-206.

9. Nelson LM, Covington SN, Rebar RW, et al. An update: spontaneous premature ovarian failure is not an early menopause Fertil Steril 2005; 83: 1327-1332.

10. Lakhal B, Braham R, Berguigua R, et al. Cytogenetic analyses of premature ovarian failure using karyotyping and interphase fluorescence in situ hybridization (FISH) in a group of 1000 patients. Clin Genet 2010; 78: 181-185.

11. Cordts EB, Christofolini DM, Dos Santos AA, et al. Genetic aspects of premature ovarian failure: a literature review. Arch Gynecol Obstet 2011; 283: 635-643.

12. Jin $M, Y u ~ Y$, Huang $H$. An update on primary ovarian insufficiency. Sci China Life Sci 2012; 55: 677-686.

13. Ebrahimi M, Akbari Asbagh F. Pathogenesis and Causes of Premature Ovarian Failure: An Update. Int J Fertil Steril 2011; 5: 54-65.

14. Goswami D, Conway GS. Premature ovarian failure. Hum Reprod Update 2005; 11: 391-410.

15. Sullivan AK, Marcus M, Epstein MP, et al. Association of FMR1 repeat size with ovarian dysfunction. Hum Reprod 2005; 20: 402-412.

16. Fortuño C, Labarta E. Genetics of primary ovarian insufficiency: a review. J Assist Reprod Genet 2014; 31: 1573-1585.

17. Otsuka F, McTavish K, Shimasaki S. Integral Role of GDF-9 and BMP-15 in Ovarian Function. Mol Reprod Dev 2011; 78: 9-21.

18. Spicer LJ, Aad PY, Allen DT, et al. Growth differentiation factor 9 (GDF9) stimulates proliferation and inhibits steroidogenesis by bovine theca cells: influence of follicle size on responses to GDF9. Biol Reprod 2008; 78: 243-253.

19. La Marca A, Brozzetti A, Sighinolfi G, et al. Primary ovarian insufficiency: autoimmune causes. Curr Opin Obstet Gynecol 2010; 22: 277-282.

20. Dittmar M, Kahaly GJ. Polyglandular autoimmune syndromes: immunogenetics and long-term follow up. J Clin Endocrinol Metab 2003; 88: 2983-2992.

21. Silva CA, Yamakami LY, Aikawa NE, et al. Autoimmune primary ovarian insufficiency. Autoimmun Rev 2014; 13: 427-430.

22. Fridovich-Keil JL, Gubbels CS, Spencer JB, et al. Ovarian function in girls and women with GALT-deficiency galactosemia. J Inherit Metab Dis 2011; 34: 357-366.

23. Auchus RJ. Steroid 17-Hydroxylase and 17,20-Lyase Deficiencies, Genetic and Pharmacologic. J Steroid Bioch Mol Biol 2017; 165 (Pt A): 71-78.

24. Larsen EC, Muller J, Schmiegelow K, et al. Reduced ovarian function in long - term survivors of radiation -and chemotherapy treated childhood cancer. J Clin Endocrinol Metab 2003; 88: 5307-5314.

25. Somigliana E, Berlanda N, Benaglia L, et al. Surgical excision of endometriomas and ovarian reserve: a systematic on serum antymullerian hormone level modifications. Fertil Steril 2012; 98: 1531-1538.

26. Kokcu A. Premature ovarian failure from current perspective. Gynecol Endocrinol 2010; 26: 555-562.

27. Vabre P, Gatimel N, Moreau J, et al. Environmental pollutants, a possible etiology for premature ovarian insufficiency: a narrative review of animal and human data. Environ Health 2017; 16: 37.

28. Bachelot A, Rouxel A, Massin N, et al. Phenotyping and genetic studies of 357 consecutive patients presenting with premature ovarian failure. Eur J Endocrinol 2009; 161: 179-187.

29. Rebar RW, Connolly HV. Clinical features of young women with hypergonadotropic amenorrhea. Fertil Steril 1990; 53: 804-810.

30. Kovanci E, Schutt AK. Premature ovarian failure: clinical presentation and treatment. Obstet Gynecol Clin North Am 2015; 42: 153-161.

31. Rocha VB, Guerra-Junior G, Marques-de-Faria AP, et al. Complete gonadal dysgenesis in clinical practice: the $46 \mathrm{XY}$ karyotype accounts for more than one third of cases Fertil Steril 2011; 96: 1431-1434.

32. Chitayat D, Wyatt PR, Wilson RD, et al. Genetics Committee of the Society of Obstetricians and Gynaecologist of Canada, Prenatal Diagnosis Committee of the canadaian College of Medical genetics. Fragile X testing in Obstetrics and Gynaecology in Canada. J Obstet Gynaecol Can 2008; 30: 837-846. 